\title{
Certain mean values and non-vanishing of automorphic $L$-functions with large level
}

\author{
by \\ Yuichi KamiYa (Nagoya)
}

1. Introduction. Let $N$ and $q$ be positive integers, and $k$ a positive even integer throughout. We denote by $S_{k}\left(\Gamma_{0}(N)\right)$ the space of all holomorphic cusp forms of weight $k$ with respect to $\Gamma_{0}(N)$. For a Dirichlet character $\chi(\bmod q)$ and $f(z)=\sum_{n=1}^{\infty} \widehat{a}_{f, \infty}(n) e^{2 \pi i n z} \in S_{k}\left(\Gamma_{0}(N)\right)$ the twisted $L$ function is defined by

$$
L(f, \chi, s)=\sum_{n=1}^{\infty} \frac{\chi(n) a_{f, \infty}(n)}{n^{s}},
$$

where $a_{f, \infty}(n)=\widehat{a}_{f, \infty}(n) n^{-(k-1) / 2}$ and $s=\sigma+i t$ is a complex variable. The above infinite series is absolutely convergent for $\sigma>1$. If $\chi$ is a primitive character $(\bmod q)$ with $(N, q)=1$, the function $L(f, \chi, s)$ can be holomorphically continued to the whole $s$-plane and satisfies a functional equation.

It is important to study the vanishing order of $L(f, \chi, s)$ at $s=1 / 2$ which is the center of the functional equation. Here we quote the following non-vanishing result due to Duke [3].

THEOREM 1 (Duke). Let $p$ be a prime and $\chi$ a primitive character (mod $q)$ with $(p, q)=1$. Let $\mathfrak{F}_{p}$ denote an orthogonal basis in $S_{2}\left(\Gamma_{0}(p)\right)$ which consists of normalized newforms. Then there is a positive absolute constant $C$ and a constant $C_{q}$ depending only on $q$ such that for $p>C_{q}$ there are at least $C p(\log p)^{-2}$ forms $f \in \mathfrak{F}_{p}$ for which $L(f, \chi, 1 / 2) \neq 0$.

The proof of the above result is based on a comparison of mean values and an important estimate concerning the Petersson norm of $f$ due to HoffsteinLockhart [6] and Goldfeld-Hoffstein-Lieman [5].

The purpose of this paper is to derive a non-vanishing result on the critical line $s=1 / 2+i t$ corresponding to Theorem 1 . To attain this purpose,

2000 Mathematics Subject Classification: Primary 11M41.

The author was supported by JSPS Research Fellowships for Young Scientists. 
first we shall give the following result which is a consequence of a comparison of certain mean values.

Theorem 2. Let $k$ be a positive even integer, $N \geq 2, \tau=|t|+2$, and $\chi$ a primitive character $(\bmod q)$ with $(N, q)=1$. Let $\mathcal{F}$ be an orthonormal basis in $S_{k}\left(\Gamma_{0}(N)\right)$. In the case $k=2$ choose $N$ with

$$
\frac{\sqrt{N}}{(\log (N+1))^{2}} \geq \max \left\{q \tau(\log (q \tau))^{2}, C_{2}\right\},
$$

and for $k>2$ choose $N$ with

$$
\frac{\sqrt{N}}{\log (N+1)} \geq \max \left\{q \tau \log (q \tau), C_{k}\right\},
$$

where $C_{k}$ is a positive constant depending only on $k$. Then, for a positive absolute constant $\mathcal{C}$, we have

$$
\sum_{\substack{f \in \mathcal{F} \\ L(f, \chi, 1 / 2+i t) \neq 0}}\left|a_{f, \infty}(1)\right|^{2} \geq \frac{(4 \pi)^{k-1}}{(k-2) !} \cdot \frac{\mathcal{C}}{\log N} .
$$

This result is an analogue of Proposition 3 of Duke [3], but we put an emphasis on the conditions for lower bounds of $N$. These conditions are necessary because the dependence on $q$ and $\tau$ appearing in estimating mean values of $L(f, \chi, 1 / 2+i t)$ should be taken into consideration. If we apply the method of Duke [3] to estimate mean values, we cannot obtain satisfactory results with respect to $q$ and $\tau$ in the error term (see the remark after the proof of Proposition 15). Thus we shall use another approach, that is, the method of Balasubramanian-Ramachandra which appears in their argument for estimating mean squares of Dirichlet $L$-functions (see the proof of Lemma $2^{\prime}$ of [1]), and obtain certain mean value results for $L(f, \chi, 1 / 2+i t)$ whose dependence on $q$ and $\tau$ is satisfactorily estimated (see Propositions 7 and 10 in Sections 3 and 4).

It should be noted that, from Propositions 1.40 and 1.43 and Theorem 2.24 of Shimura [9], it follows that

$$
\operatorname{dim} S_{k}\left(\Gamma_{0}(N)\right)=\frac{(k-1) N}{12} \prod_{p \mid N}(1+1 / p)+O\left(N^{1 / 2} d(N)\right)
$$

for $N \geq c$, where $c$ is a positive absolute constant, $p$ is a prime, $d(\cdot)$ is the divisor function, and the implied constant is absolute. Hence we see that $S_{k}\left(\Gamma_{0}(N)\right) \neq\{0\}$ for $N \geq c$.

It seems difficult to obtain an upper bound for $a_{f, \infty}(1)$ as $N$ and $f \in$ $\mathcal{F}$ vary. For $k=2,4,6,8,10,14$ and $N$ a large prime, $S_{k}\left(\Gamma_{0}(N)\right)$ has an orthonormal basis which consists of newforms. Hence, in these cases, we can 
apply an upper bound for $a_{f, \infty}(1)$ proved in [6] and [5] to Theorem 2, and give the following variation of Theorem 1 .

TheOREM 3. Let $p$ be a prime, $\chi$ a primitive character $(\bmod q)$ with $(p, q)=1$, and $\tau=|t|+2$. For $k=2,4,6,8,10,14$ let $\mathfrak{F}_{k, p}$ denote an orthogonal basis in $S_{k}\left(\Gamma_{0}(p)\right)$ which consists of normalized newforms. In the case $k=2$ choose $p$ with

$$
\frac{\sqrt{p}}{(\log (p+1))^{2}} \geq \max \left\{q \tau(\log (q \tau))^{2}, C_{2}^{\prime}\right\}
$$

and for $k=4,6,8,10,14$ choose $p$ with

$$
\frac{\sqrt{p}}{\log (p+1)} \geq \max \left\{q \tau \log (q \tau), C_{k}^{\prime}\right\}
$$

where $C_{k}^{\prime}$ is a positive constant depending only on $k$. Then, for a positive absolute constant $\mathcal{C}^{\prime}$, there are at least $\mathcal{C}^{\prime} p(\log p)^{-2}$ forms $f \in \mathfrak{F}_{k, p}$ for which $L(f, \chi, 1 / 2+i t) \neq 0$.

In the last section we shall consider an asymptotic formula for the sum $\sum_{f \in \mathcal{F}}|L(f, \chi, 1 / 2+i t)|^{2}$ by generalizing the method of Duke [3] (see Proposition 15 in Section 6).

The author would like to express his sincere gratitude to Professor Kohji Matsumoto and Professor Yoshio Tanigawa for their advice and encouragement. He would also like to express his sincere thanks to Professor Fernando Chamizo and the referee for their helpful comments.

2. Preliminaries. In this section, following Iwaniec's book [7], we introduce notation and known results.

First, we refer to Kloosterman sums. Let $\Gamma$ be a Fuchsian group of the first kind which contains parabolic motions. Select a complete set of inequivalent cusps for $\Gamma$, and let $\mathfrak{a}, \mathfrak{b}$ be elements in that set (not necessarily distinct). We denote by $\sigma_{\mathfrak{a}}, \sigma_{\mathfrak{b}} \in \mathrm{SL}_{2}(\mathbb{R})$ the scaling matrices with respect to $\mathfrak{a}, \mathfrak{b}$. Put

$$
\mathcal{C}(\mathfrak{a}, \mathfrak{b})=\mathcal{C}_{\Gamma}(\mathfrak{a}, \mathfrak{b})=\left\{c>0 \mid\left(\begin{array}{ll}
* & * \\
c & *
\end{array}\right) \in \sigma_{\mathfrak{a}}^{-1} \Gamma \sigma_{\mathfrak{b}}\right\}
$$

and

$$
B=\left\{ \pm\left(\begin{array}{ll}
1 & b \\
0 & 1
\end{array}\right) \mid b \in \mathbb{Z}\right\}
$$

For integers $m, n$ and $c \in \mathcal{C}(\mathfrak{a}, \mathfrak{b})$ the Kloosterman sum is defined by

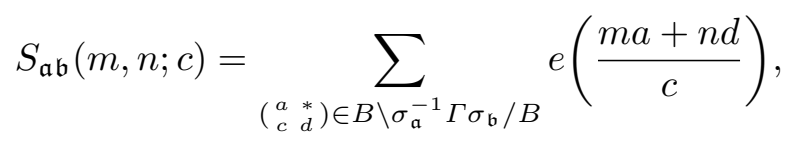


where $e(\cdot)=e^{2 \pi i \cdot}$. In particular, set

$$
S(m, n ; c)=\sum_{a d \equiv 1(\bmod c)} e\left(\frac{m a+n d}{c}\right) .
$$

We are interested in the case $\Gamma=\Gamma_{0}(N)$ with $N \geq 2$ and $\mathfrak{a}=\infty, \mathfrak{b}=0$. In this case one can show the following properties:

$$
\begin{aligned}
& \sigma_{\infty}=\left(\begin{array}{ll}
1 & 0 \\
0 & 1
\end{array}\right), \quad \sigma_{0}=\left(\begin{array}{cc}
0 & -1 / \sqrt{N} \\
\sqrt{N} & 0
\end{array}\right), \\
& \mathcal{C}(\infty, \infty)=\mathcal{C}(0,0)=\{r N \mid r \in \mathbb{N}\}, \\
& \mathcal{C}(\infty, 0)=\mathcal{C}(0, \infty)=\{r \sqrt{N} \mid r \in \mathbb{N},(r, N)=1\}, \\
& S_{\infty \infty}(m, n ; c)=S_{00}(m, n ; c)=S(m, n ; c), \\
& S_{\infty 0}(m, n ; c)=S_{0 \infty}(m, n ; c)=S(m \bar{N}, n ; c / \sqrt{N}),
\end{aligned}
$$

where $\bar{N}$ is the residue class with $\bar{N} N \equiv 1(\bmod c / \sqrt{N})($ see p. 58 of [7]).

Next, we mention the functional equation for $L(f, \chi, s)$. For $f \in$ $S_{k}\left(\Gamma_{0}(N)\right)$ and $\gamma \in \mathrm{SL}_{2}(\mathbb{R})$ we define the function $f \mid[\gamma]_{k}$ by

$$
\left(f \mid[\gamma]_{k}\right)(z)=(c z+d)^{-k} f\left(\frac{a z+b}{c z+d}\right), \quad \gamma=\left(\begin{array}{ll}
a & b \\
c & d
\end{array}\right) .
$$

If $\chi$ is a primitive character $(\bmod q)$ with $(N, q)=1$, the function $L(f, \chi, s)$ can be holomorphically continued to the whole $s$-plane and satisfies the functional equation (see Theorem 7.6 of [7])

$$
\begin{aligned}
\left(\frac{2 \pi}{\sqrt{N} q}\right)^{-s} \Gamma & (s+(k-1) / 2) L(f, \chi, s) \\
& =\mu\left(\frac{2 \pi}{\sqrt{N} q}\right)^{s-1} \Gamma((k+1) / 2-s) L\left(f \mid\left[\sigma_{0}\right]_{k}, \bar{\chi}, 1-s\right)
\end{aligned}
$$

where $\mu=i^{k} \chi(N) W(\chi)^{2} q^{-1}, W(\chi)$ is the Gauss sum, and $\sigma_{0}$ is the same as in (1) for $N \geq 2$ and $\sigma_{0}$ denotes the unit matrix for $N=1$. Recall that $f \mid\left[\sigma_{0}\right]_{k}$ is also an element in $S_{k}\left(\Gamma_{0}(N)\right)$.

Finally, we quote the Petersson formula. In the finite linear space $S_{k}\left(\Gamma_{0}(N)\right)$ the Petersson inner product is defined by

$$
\langle f, g\rangle=\int_{\Gamma_{0}(N) \backslash \mathfrak{H}} f(z) \overline{g(z)} y^{k} d \mu(z),
$$

where $f, g \in S_{k}\left(\Gamma_{0}(N)\right), \mathfrak{H}$ is the upper half-plane, $z=x+i y$, and $d \mu(z)=$ $y^{-2} d x d y$. We denote by $\mathcal{F}$ an orthonormal basis in $S_{k}\left(\Gamma_{0}(N)\right)$.

Theorem 4 (Petersson formula). Let $k$ be a positive even integer, $\mathcal{F}$ an orthonormal basis in $S_{k}\left(\Gamma_{0}(N)\right)$, and let $\mathfrak{a}, \mathfrak{b}$ be cusps for $\Gamma_{0}(N)$. For 
$f \in S_{k}\left(\Gamma_{0}(N)\right)$ and a scaling matrix $\sigma_{\mathfrak{a}}$ we expand $f \mid\left[\sigma_{\mathfrak{a}}\right]_{k}$ as

$$
\left(f \mid\left[\sigma_{\mathfrak{a}}\right]_{k}\right)(z)=\sum_{n=1}^{\infty} \widehat{a}_{f, \mathfrak{a}}(n) e^{2 \pi i n z}
$$

and we define $a_{f, \mathfrak{a}}(n)=\widehat{a}_{f, \mathfrak{a}}(n) n^{-(k-1) / 2}$. Then, for any positive integers $m, n$, we have

$$
\begin{aligned}
& \frac{(k-2) !}{(4 \pi)^{k-1}} \sum_{f \in \mathcal{F}} \overline{a_{f, \mathfrak{a}}(m)} a_{f, \mathfrak{b}}(n) \\
& \quad=\delta_{m n} \delta_{\mathfrak{a} \mathfrak{b}}+2 \pi i^{-k} \sum_{c \in \mathcal{C}(\mathfrak{a}, \mathfrak{b})} c^{-1} S_{\mathfrak{a} \mathfrak{b}}(m, n ; c) J_{k-1}\left(\frac{4 \pi \sqrt{m n}}{c}\right),
\end{aligned}
$$

where $J_{k-1}(\cdot)$ is the Bessel function of order $k-1$, and $\delta_{m n}$ and $\delta_{\mathfrak{a} \mathfrak{b}}$ are (respectively) defined by

$$
\delta_{m n}=\left\{\begin{array}{ll}
1 & \text { if } m=n, \\
0 & \text { if } m \neq n,
\end{array} \quad \delta_{\mathfrak{a} \mathfrak{b}}= \begin{cases}1 & \text { if } \mathfrak{a}=\mathfrak{b} \\
0 & \text { if } \mathfrak{a} \neq \mathfrak{b} .\end{cases}\right.
$$

For the proof of the above theorem see p. 54 of [7].

3. An asymptotic formula for the first moment. In this section we shall derive an asymptotic formula for the first moment which consists of averaging the value $\overline{a_{f, \infty}(1)} L(f, \chi, 1 / 2+i t)$ over $\mathcal{F}$.

First, we prove the following lemma by modifying the proof of Lemma 1 of Duke [3].

LEMma 5. Let $k$ be a positive even integer and $\mathcal{F}$ an orthonormal basis in $S_{k}\left(\Gamma_{0}(N)\right)$.

(i) For $N \geq 1$ we have

$\left|\frac{(k-2) !}{(4 \pi)^{k-1}} \sum_{f \in \mathcal{F}} \overline{a_{f, \infty}(m)} a_{f, \infty}(n)-\delta_{m n}\right| \ll(m, n)^{1 / 2}(m n)^{(k-1) / 2} N^{1 / 2-k} d(N)$

and

$\left|\frac{(k-2) !}{(4 \pi)^{k-1}} \sum_{f \in \mathcal{F}} \overline{a_{f, 0}(m)} a_{f, 0}(n)-\delta_{m n}\right| \ll(m, n)^{1 / 2}(m n)^{(k-1) / 2} N^{1 / 2-k} d(N)$,

where $d(\cdot)$ is the divisor function, and the implied constants depend only on $k$.

(ii) For $N \geq 2$ we have

$$
\left|\frac{(k-2) !}{(4 \pi)^{k-1}} \sum_{f \in \mathcal{F}} \overline{a_{f, \infty}(1)} a_{f, 0}(n)\right| \ll n^{(k-1) / 2} N^{-k / 2},
$$

where the implied constant depends only on $k$. 
Proof. The estimates in (i) are proved by the same argument as in the proof of Lemma 1 of [3]. The quantities on the left-hand sides in (i) are equal to

$$
\left|2 \pi i^{-k} \sum_{\substack{c>0 \\ c \equiv 0(\bmod N)}} c^{-1} S(m, n ; c) J_{k-1}\left(\frac{4 \pi \sqrt{m n}}{c}\right)\right|
$$

by Theorem 4, (2), and (4). By using Weil's bound (see [10])

$$
|S(m, n ; c)| \leq(m, n, c)^{1 / 2} c^{1 / 2} d(c)
$$

and the estimate

$$
J_{k-1}(y) \ll y^{k-1}
$$

for $y \geq 0$, where the implied constant depends on $k$, the quantity ( 7$)$ is

$$
\ll(m, n)^{1 / 2}(m n)^{(k-1) / 2} \sum_{\substack{c>0 \\ c \equiv 0(\bmod N)}} c^{1 / 2-k} d(c) .
$$

Putting $c=r N$ and using $d(c) \leq d(r) d(N)$, we obtain the estimates in (i).

The quantity on the left-hand side in (ii) is equal to

$$
\left|2 \pi i^{-k} \sum_{c \in \mathcal{C}(\infty, 0)} c^{-1} S(\bar{N}, n ; c / \sqrt{N}) J_{k-1}\left(\frac{4 \pi \sqrt{n}}{c}\right)\right|
$$

by Theorem 4, (5), and the fact that the cusps $\infty, 0$ are inequivalent for $N \geq 2$. By using Weil's bound and (8), we see that (9) is

$$
\ll \sum_{c \in \mathcal{C}(\infty, 0)} c^{-1}(\bar{N}, n, c / \sqrt{N})^{1 / 2}(c / \sqrt{N})^{1 / 2} d(c / \sqrt{N})(\sqrt{n} / c)^{k-1},
$$

and by noting $(\bar{N}, c / \sqrt{N})=1$, this is

$$
\ll \sum_{c \in \mathcal{C}(\infty, 0)} c^{-1}(c / \sqrt{N})^{1 / 2} d(c / \sqrt{N})(\sqrt{n} / c)^{k-1} .
$$

Putting $c=r \sqrt{N}$ (see (3)), we obtain the estimate in (ii).

Lemma 6. Let $f \in S_{k}\left(\Gamma_{0}(N)\right)$ and $\chi$ a primitive character $(\bmod q)$ with $(N, q)=1$. Let $l$ be a positive integer, $x, y \geq 1,1 / 2<\alpha<l, 0<\beta<1 / 2$, and $0<\gamma<l$. Then 


$$
\begin{aligned}
L(f, \chi, 1 / 2+i t)= & \sum_{n \leq x} \frac{\chi(n) a_{f, \infty}(n)}{n^{1 / 2+i t}} \\
& +\mu\left(\frac{2 \pi}{\sqrt{N} q}\right)^{2 i t} \frac{\Gamma(k / 2-i t)}{\Gamma(k / 2+i t)} \sum_{n \leq y} \frac{\overline{\chi(n)} a_{f, 0}(n)}{n^{1 / 2-i t}} \\
& +I_{1, f}+I_{2, f}-\frac{\mu}{2 \pi i}\left(\frac{2 \pi}{\sqrt{N} q}\right)^{2 i t}\left(I_{3, f}+I_{4, f}\right)
\end{aligned}
$$

with

$$
\begin{aligned}
& I_{1, f}=\sum_{n>x} \frac{\chi(n) a_{f, \infty}(n)}{n^{1 / 2+i t}} e^{-(n / x)^{l}}, \\
& I_{2, f}=\frac{1}{2 \pi i} \int_{(-\gamma)} x^{w} \frac{\Gamma(1+w / l)}{w} \sum_{n \leq x} \frac{\chi(n) a_{f, \infty}(n)}{n^{1 / 2+i t+w}} d w, \\
& I_{3, f}=\int_{(\beta)}\left(\frac{4 \pi^{2} x}{N q^{2}}\right)^{w} \frac{\Gamma(k / 2-i t-w)}{\Gamma(k / 2+i t+w)} \cdot \frac{\Gamma(1+w / l)}{w} \sum_{n \leq y} \frac{\overline{\chi(n)} a_{f, 0}(n)}{n^{1 / 2-i t-w}} d w, \\
& I_{4, f}=\int_{(-\alpha)}\left(\frac{4 \pi^{2} x}{N q^{2}}\right)^{w} \frac{\Gamma(k / 2-i t-w)}{\Gamma(k / 2+i t+w)} \cdot \frac{\Gamma(1+w / l)}{w} \sum_{n>y} \frac{\overline{\chi(n)} a_{f, 0}(n)}{n^{1 / 2-i t-w}} d w,
\end{aligned}
$$

where $\int_{(c)}$ denotes the integral along the vertical line $w=c+i v,-\infty<v<\infty$.

Proof. This lemma is a modification of Lemma $1^{\prime}$ of [1], hence it is sufficient to give a brief sketch.

Since, for $X>0$,

$$
e^{-X^{l}}=\frac{1}{2 \pi i} \int_{(1)} X^{-w} \frac{\Gamma(1+w / l)}{w} d w,
$$

we have

$$
\begin{aligned}
\sum_{n=1}^{\infty} \frac{\chi(n) a_{f, \infty}(n)}{n^{1 / 2+i t}} e^{-(n / x)^{l}} & \\
& =\frac{1}{2 \pi i} \int_{(1)} L(f, \chi, 1 / 2+i t+w) x^{w} \frac{\Gamma(1+w / l)}{w} d w .
\end{aligned}
$$

We move the contour of integration to the vertical line $\Re w=-\alpha$, and calculate the residue of the integrand at $w=0$. Next, we use the functional equation (6) to express $L(f, \chi, 1 / 2+i t+w)$ by the form involving the infinite series, and divide this infinite series into two parts $n \leq y$ and $n>y$. We move the contour of integration involving the finite sum over $n \leq y$ to the vertical line $\Re w=\beta$, and calculate the residue of the integrand at $w=0$. 
Finally, noting the equation

$$
e^{-X^{l}}=1+\frac{1}{2 \pi i} \int_{(-\gamma)} X^{-w} \frac{\Gamma(1+w / l)}{w} d w,
$$

we obtain the equation of the lemma.

Proposition 7. Let $k$ be a positive even integer, $N \geq 2, \tau=|t|+2$, $\mathcal{F}$ an orthonormal basis in $S_{k}\left(\Gamma_{0}(N)\right)$, and $\chi$ a primitive character $(\bmod q)$ with $(N, q)=1$. Then

$$
\sum_{f \in \mathcal{F}} \overline{a_{f, \infty}(1)} L(f, \chi, 1 / 2+i t)=\frac{(4 \pi)^{k-1}}{(k-2) !}+O\left(\left(\frac{q \tau}{\sqrt{N}}\right)^{k / 2}\right),
$$

where the implied constant depends only on $k$.

Proof. To estimate the first moment, multiply the quantities on the right-hand side of the formula of Lemma 6 by $\overline{a_{f, \infty}(1)}$ and sum these over $\mathcal{F}$. Then estimate each sum separately. For technical reasons, we restrict $l=k$ in the formula of Lemma 6 . Recall that $x, y \geq 1, l$ is a positive integer, and $1 / 2<\alpha<l, 0<\beta<1 / 2,0<\gamma<l$. We will shortly choose these values to optimize the error terms.

First, by Lemma 5(ii) and Stirling's formula, we have

$$
\begin{aligned}
\sum_{f \in \mathcal{F}} \overline{a_{f, \infty}(1)} I_{3, f} \ll & \left(\frac{x}{N q^{2}}\right)^{\beta} \sum_{n \leq y} n^{\beta-1 / 2}\left|\sum_{f \in \mathcal{F}} \overline{a_{f, \infty}(1)} a_{f, 0}(n)\right| \\
& \times \int_{(\beta)}\left|\frac{\Gamma(k / 2-i t-w)}{\Gamma(k / 2+i t+w)} \cdot \frac{\Gamma(1+w / k)}{w}\right||d w| \\
\ll & \left(\frac{x y}{N q^{2}}\right)^{\beta}\left(\frac{y}{N}\right)^{k / 2} \\
& \times \int_{(\beta)}\left|\frac{\Gamma(k / 2-i t-w)}{\Gamma(k / 2+i t+w)} \cdot \frac{\Gamma(1+w / k)}{w}\right||d w| \\
\ll & \left(\frac{x y}{N q^{2} \tau^{2}}\right)^{\beta}\left(\frac{y}{N}\right)^{k / 2} .
\end{aligned}
$$

Second, by Lemma 5(ii), we have

$$
\begin{aligned}
\sum_{f \in \mathcal{F}} \overline{a_{f, \infty}(1)} I_{4, f} \ll & \left(\frac{x}{N q^{2}}\right)^{-\alpha} N^{-k / 2} \sum_{n>y} n^{k / 2-\alpha-1} \\
& \times \int_{(-\alpha)}\left|\frac{\Gamma(k / 2-i t-w)}{\Gamma(k / 2+i t+w)} \cdot \frac{\Gamma(1+w / k)}{w}\right||d w| .
\end{aligned}
$$


Now we fix $\alpha$ with $k / 2<\alpha<k$ to get

$$
\sum_{n>y} n^{k / 2-\alpha-1} \ll y^{k / 2-\alpha} .
$$

Hence, by Stirling's formula, we have

$$
\sum_{f \in \mathcal{F}} \overline{a_{f, \infty}(1)} I_{4, f} \ll\left(\frac{x y}{N q^{2} \tau^{2}}\right)^{-\alpha}\left(\frac{y}{N}\right)^{k / 2} .
$$

Third, by Lemma 5(i) and Stirling's formula, we have

$$
\begin{aligned}
\sum_{f \in \mathcal{F}} \overline{a_{f, \infty}(1)} I_{2, f} \ll & x^{-\gamma} \sum_{n \leq x} n^{\gamma-1 / 2}\left(\delta_{1 n}+n^{(k-1) / 2} N^{1 / 2-k} d(N)\right) \\
& \times \int_{(-\gamma)}\left|\frac{\Gamma(1+w / k)}{w}\right||d w| \\
\ll & x^{-\gamma}+N^{1 / 2-k} d(N) x^{k / 2} .
\end{aligned}
$$

Fourth, by Lemma 5(i), we have

$$
\begin{aligned}
\sum_{f \in \mathcal{F}} \overline{a_{f, \infty}(1)} I_{1, f} & \ll N^{1 / 2-k} d(N) \sum_{n>x} n^{k / 2-1} e^{-(n / x)^{k}} \\
& \ll N^{1 / 2-k} d(N) x^{k / 2} .
\end{aligned}
$$

Fifth, by Lemma 5(ii), we have

$$
\sum_{f \in \mathcal{F}} \overline{a_{f, \infty}(1)} \sum_{n \leq y} \frac{\overline{\chi(n)} a_{f, 0}(n)}{n^{1 / 2-i t}} \ll N^{-k / 2} y^{k / 2} .
$$

Finally, by Lemma 5(i), we have

$$
\begin{aligned}
\sum_{f \in \mathcal{F}} \overline{a_{f, \infty}(1)} \sum_{n \leq x} \frac{\chi(n) a_{f, \infty}(n)}{n^{1 / 2+i t}} \\
=\sum_{n \leq x} \frac{\chi(n)}{n^{1 / 2+i t}}\left\{\frac{(4 \pi)^{k-1}}{(k-2) !} \delta_{1 n}+O\left(n^{(k-1) / 2} N^{1 / 2-k} d(N)\right)\right\} \\
=\frac{(4 \pi)^{k-1}}{(k-2) !}+O\left(N^{1 / 2-k} d(N) x^{k / 2}\right) .
\end{aligned}
$$

Specifying $x=y=\sqrt{N} q \tau$ and $\gamma=k / 2$ in (10)-(15), we obtain the assertion of this proposition.

Corollary 8. We use the same notation as in Proposition 7. Choose $N$ with

$$
\frac{\sqrt{N}}{\log (N+1)} \geq \max \left\{q \tau, C_{1, k}\right\}
$$


where $C_{1, k}$ is a positive constant depending only on $k$. Then

$$
\left|\sum_{f \in \mathcal{F}} \overline{a_{f, \infty}(1)} L(f, \chi, 1 / 2+i t)\right| \geq \frac{1}{2} \cdot \frac{(4 \pi)^{k-1}}{(k-2) !} .
$$

Pr o of. From Proposition 7 it follows that

$$
\left|\sum_{f \in \mathcal{F}} \overline{a_{f, \infty}(1)} L(f, \chi, 1 / 2+i t)\right| \geq \frac{(4 \pi)^{k-1}}{(k-2) !}-O\left(\left(\frac{q \tau}{\sqrt{N}}\right)^{k / 2}\right) .
$$

If we choose $N$ satisfying

$$
\frac{\sqrt{N}}{\log (N+1)} \geq \max \left\{q \tau, C_{1, k}\right\}
$$

for an appropriate constant $C_{1, k}$, then the error term on the right-hand side is $o(1)$. This completes the proof.

4. An upper bound for the second moment. In this section we shall derive an upper bound for the second moment which consists of averaging the value $|L(f, \chi, 1 / 2+i t)|^{2}$ over $\mathcal{F}$. To estimate the second moment we use the method of Balasubramanian-Ramachandra which is useful to observe the dependence on $q$ and $\tau$ (see the proof of Lemma $2^{\prime}$ of [1]), and use the following important result due to Duke-Friedlander-Iwaniec [4] and Iwaniec [7].

Theorem 9 (Duke-Friedlander-Iwaniec and Iwaniec). Let $k$ be a positive even integer, $x \geq 1$, and $\mathcal{F}$ an orthonormal basis in $S_{k}\left(\Gamma_{0}(N)\right)$. Then, for any complex numbers $b_{n}$, we have

$$
\frac{(k-2) !}{(4 \pi)^{k-1}} \sum_{f \in \mathcal{F}}\left|\sum_{n \leq x} b_{n} a_{f, \infty}(n)\right|^{2}=\{1+O(\varphi(x))\} \sum_{n \leq x}\left|b_{n}\right|^{2}
$$

with

$$
\varphi(x)= \begin{cases}\frac{x \log (x+1)}{N} & \text { if } k=2, \\ \frac{x}{N} & \text { if } k>2,\end{cases}
$$

where the implied constant is absolute.

For the result in the case $k=2$ see Theorem 1 of [4], and in the case $k>2$ see Theorem 5.7 of [7]. This result is proved by using Theorem 4 . The formula obtained by replacing $\infty$ by 0 on the left-hand side of (16) is also valid, because of $(2)$ and (4).

Proposition 10. Let $k$ be a positive even integer, $\tau=|t|+2, \mathcal{F}$ an orthonormal basis in $S_{k}\left(\Gamma_{0}(N)\right)$, and $\chi$ a primitive character $(\bmod q)$ with $(N, q)=1$. Then 


$$
\begin{aligned}
L(f, \chi, 1 / 2+i t)= & \sum_{n \leq \sqrt{N} q \tau} \frac{\chi(n) a_{f, \infty}(n)}{n^{1 / 2+i t}} \\
& +\mu\left(\frac{2 \pi}{\sqrt{N} q}\right)^{2 i t} \frac{\Gamma(k / 2-i t)}{\Gamma(k / 2+i t)} \sum_{n \leq \sqrt{N} q \tau} \frac{\overline{\chi(n)} a_{f, 0}(n)}{n^{1 / 2-i t}} \\
& +R(f, N, q, t)
\end{aligned}
$$

with

$$
\sum_{f \in \mathcal{F}}|R(f, N, q, t)|^{2} \ll \begin{cases}1+\frac{q \tau \log (\sqrt{N} q \tau)}{\sqrt{N}} & \text { if } k=2, \\ 1+\frac{q \tau}{\sqrt{N}} & \text { if } k>2,\end{cases}
$$

where the implied constant depends only on $k$.

Proof. We put $l=2$ in the formula of Lemma 6 , and set

$$
R_{f}(x, y)=I_{1, f}+I_{2, f}-\frac{\mu}{2 \pi i}\left(\frac{2 \pi}{\sqrt{N} q}\right)^{2 i t}\left(I_{3, f}+I_{4, f}\right) .
$$

We shall estimate $\sum_{f \in \mathcal{F}}\left|R_{f}(x, y)\right|^{2}$. Since

$$
\sum_{f \in \mathcal{F}}\left|R_{f}(x, y)\right|^{2} \leq 4 \sum_{h=1}^{4} \sum_{f \in \mathcal{F}}\left|I_{h, f}\right|^{2},
$$

it is sufficient to estimate each sum on the right-hand side separately. Note that the conditions on $x, y, \beta$ are as in Lemma 6 but those on $\alpha, \gamma$ are now $1 / 2<\alpha<2,0<\gamma<2$, respectively. Later in the proof we will restrict the range of $\alpha$ to $1<\alpha<2$ and specify $x=y=\sqrt{N} q \tau$.

First, by Cauchy's inequality, Theorem 9, and Stirling's formula, we have

$$
\begin{aligned}
\sum_{f \in \mathcal{F}}\left|I_{3, f}\right|^{2} \leq & \int_{(\beta)}\left|\frac{\Gamma(k / 2-i t-w)}{\Gamma(k / 2+i t+w)} \cdot \frac{\Gamma(1+w / 2)}{w}\right||d w| \\
& \times \int_{(\beta)}\left(\frac{4 \pi^{2} x}{N q^{2}}\right)^{2 \beta}\left|\frac{\Gamma(k / 2-i t-w)}{\Gamma(k / 2+i t+w)} \cdot \frac{\Gamma(1+w / 2)}{w}\right| \\
& \times \sum_{f \in \mathcal{F}}\left|\sum_{n \leq y} \frac{\overline{\chi(n)} a_{f, 0}(n)}{n^{1 / 2-i t-w}}\right|^{2}|d w| \\
\ll & \left(\frac{x}{N q^{2}}\right)^{2 \beta}(1+\varphi(y)) \sum_{n \leq y} n^{2 \beta-1} \\
& \times\left(\int_{(\beta)}\left|\frac{\Gamma(k / 2-i t-w)}{\Gamma(k / 2+i t+w)} \cdot \frac{\Gamma(1+w / 2)}{w}\right||d w|\right)^{2}
\end{aligned}
$$




$$
\ll\left(\frac{x y}{N q^{2} \tau^{2}}\right)^{2 \beta}(1+\varphi(y)) .
$$

Next, we estimate $\sum_{f \in \mathcal{F}}\left|I_{4, f}\right|^{2}$. We follow the method similar to that mentioned above, but we need to treat the quantity

$$
\sum_{f \in \mathcal{F}}\left|\sum_{n>y} \frac{\overline{\chi(n)} a_{f, 0}(n)}{n^{1 / 2-i t-w}}\right|^{2},
$$

where $\Re w=-\alpha$. We fix $\alpha$ with $1<\alpha<2$ to handle (19). The quantity (19) is, by Cauchy's inequality,

$$
\begin{aligned}
& \leq \sum_{f \in \mathcal{F}}\left(\sum_{j=1}^{\infty}\left|\sum_{j y<n \leq(j+1) y} \frac{\overline{\chi(n)} a_{f, 0}(n)}{n^{1 / 2-i t-w}}\right|\right)^{2} \\
& \ll \sum_{j=1}^{\infty} j^{1+\varepsilon} \sum_{f \in \mathcal{F}}\left|\sum_{j y<n \leq(j+1) y} \frac{\overline{\chi(n)} a_{f, 0}(n)}{n^{1 / 2-i t-w}}\right|^{2}
\end{aligned}
$$

for any $\varepsilon>0$. Applying Theorem 9 to the inner sum, we have

$$
\begin{aligned}
& \ll \sum_{j=1}^{\infty} j^{1+\varepsilon}\{1+\varphi((j+1) y)\} \sum_{j y<n \leq(j+1) y} n^{-2 \alpha-1} \\
& \ll(1+\varphi(y)) y^{-2 \alpha} \sum_{j=1}^{\infty} j^{1+\varepsilon^{\prime}-2 \alpha} \ll(1+\varphi(y)) y^{-2 \alpha} .
\end{aligned}
$$

Hence

$$
\sum_{f \in \mathcal{F}}\left|I_{4, f}\right|^{2} \ll\left(\frac{x y}{N q^{2} \tau^{2}}\right)^{-2 \alpha}(1+\varphi(y)) .
$$

The quantity $\sum_{f \in \mathcal{F}}\left|I_{2, f}\right|^{2}$ is estimated by an argument similar to that in the case $\sum_{f \in \mathcal{F}}\left|I_{3, f}\right|^{2}$. We have, by Cauchy's inequality, Theorem 9, and Stirling's formula,

$$
\begin{aligned}
\sum_{f \in \mathcal{F}}\left|I_{2, f}\right|^{2} & \ll x^{-2 \gamma}(1+\varphi(x)) \sum_{n \leq x} n^{2 \gamma-1}\left(\int_{(-\gamma)}\left|\frac{\Gamma(1+w / 2)}{w}\right||d w|\right)^{2} \\
& \ll 1+\varphi(x) .
\end{aligned}
$$

Finally, by an argument similar to that in the case $\sum_{f \in \mathcal{F}}\left|I_{4, f}\right|^{2}$, we have

$$
\begin{aligned}
\sum_{f \in \mathcal{F}}\left|I_{1, f}\right|^{2} & \ll \sum_{j=1}^{\infty} j^{1+\varepsilon} \sum_{f \in \mathcal{F}}\left|\sum_{j x<n \leq(j+1) x} \frac{\chi(n) a_{f, \infty}(n)}{n^{1 / 2+i t}} e^{-(n / x)^{2}}\right|^{2} \\
& \ll \sum_{j=1}^{\infty} j^{1+\varepsilon}\{1+\varphi((j+1) x)\} \sum_{j x<n \leq(j+1) x} \frac{e^{-2(n / x)^{2}}}{n}
\end{aligned}
$$




$$
\begin{aligned}
& \ll x^{4}(1+\varphi(x)) \sum_{j=1}^{\infty} j^{2+\varepsilon^{\prime \prime}} \sum_{j x<n \leq(j+1) x} \frac{1}{n^{5}} \\
& \ll 1+\varphi(x) .
\end{aligned}
$$

Specifying $x=y=\sqrt{N} q \tau$ in (17), (18), (20), (21), and (22), we obtain

$$
\sum_{f \in \mathcal{F}}|R(f, N, q, t)|^{2}=\sum_{f \in \mathcal{F}}\left|R_{f}(\sqrt{N} q \tau, \sqrt{N} q \tau)\right|^{2} \ll 1+\varphi(\sqrt{N} q \tau) .
$$

This completes the proof.

Corollary 11. With the same notation as in Proposition 10 we have

$$
\begin{aligned}
\sum_{f \in \mathcal{F}}|L(f, \chi, 1 / 2+i t)|^{2} \leq 4 \frac{(4 \pi)^{k-1}}{(k-2) !} \log (\sqrt{N} q \tau) \\
+ \begin{cases}O\left((\log (\sqrt{N} q \tau))^{1 / 2}+\frac{q \tau(\log (\sqrt{N} q \tau))^{2}}{\sqrt{N}}\right) & \text { if } k=2, \\
O\left((\log (\sqrt{N} q \tau))^{1 / 2}+\frac{q \tau \log (\sqrt{N} q \tau)}{\sqrt{N}}\right) & \text { if } k>2,\end{cases}
\end{aligned}
$$

where the implied constants depend only on $k$.

Remark. The estimate in Corollary 11 gives an improvement on Theorem 1 of Chamizo-Pomykała [2] for the case $\sigma=1$ (in their notation).

Proof. By using Cauchy's inequality, it follows that

$$
\begin{aligned}
& \sum_{f \in \mathcal{F}}|L(f, \chi, 1 / 2+i t)|^{2} \\
& \leq \sum_{f \in \mathcal{F}}\left|\sum_{n \leq \sqrt{N} q \tau} \frac{\chi(n) a_{f, \infty}(n)}{n^{1 / 2+i t}}\right|^{2}+\sum_{f \in \mathcal{F}}\left|\sum_{n \leq \sqrt{N} q \tau} \frac{\overline{\chi(n)} a_{f, 0}(n)}{n^{1 / 2-i t}}\right|^{2} \\
& +2\left(\sum_{f \in \mathcal{F}}\left|\sum_{n \leq \sqrt{N} q \tau} \frac{\chi(n) a_{f, \infty}(n)}{n^{1 / 2+i t}}\right|^{2}\right)^{1 / 2}\left(\sum_{f \in \mathcal{F}}\left|\sum_{n \leq \sqrt{N} q \tau} \frac{\overline{\chi(n)} a_{f, 0}(n)}{n^{1 / 2-i t}}\right|^{2}\right)^{1 / 2} \\
& +2\left(\sum_{f \in \mathcal{F}}|R(f, N, q, t)|^{2}\right)^{1 / 2}\left(\sum_{f \in \mathcal{F}}\left|\sum_{n \leq \sqrt{N} q \tau} \frac{\chi(n) a_{f, \infty}(n)}{n^{1 / 2+i t}}\right|^{2}\right)^{1 / 2} \\
& +2\left(\sum_{f \in \mathcal{F}}|R(f, N, q, t)|^{2}\right)^{1 / 2}\left(\sum_{f \in \mathcal{F}}\left|\sum_{n \leq \sqrt{N} q \tau} \frac{\overline{\chi(n)} a_{f, 0}(n)}{n^{1 / 2-i t}}\right|^{2}\right)^{1 / 2} \\
& +\sum_{f \in \mathcal{F}}|R(f, N, q, t)|^{2} .
\end{aligned}
$$


Here, by Theorem 9, we have

$$
\begin{aligned}
\sum_{f \in \mathcal{F}}\left|\sum_{n \leq \sqrt{N} q \tau} \frac{\chi(n) a_{f, \infty}(n)}{n^{1 / 2+i t}}\right|^{2} \\
=\left\{\frac{(4 \pi)^{k-1}}{(k-2) !}+O(\varphi(\sqrt{N} q \tau))\right\} \sum_{\substack{n \leq \sqrt{N} q \tau \\
(n, q)=1}} \frac{1}{n} \\
\leq\left\{\frac{(4 \pi)^{k-1}}{(k-2) !}+O(\varphi(\sqrt{N} q \tau))\right\}(1+\log (\sqrt{N} q \tau)) .
\end{aligned}
$$

Note that the second term on the right-hand side of (23) has the same estimate as in (24). Substituting (24) into (23), we have

$$
\begin{aligned}
\sum_{f \in \mathcal{F}}|L(f, \chi, 1 / 2+i t)|^{2} \leq & 4 \frac{(4 \pi)^{k-1}}{(k-2) !} \log (\sqrt{N} q \tau) \\
& +O\left((\log (\sqrt{N} q \tau))^{1 / 2}+\varphi(\sqrt{N} q \tau) \log (\sqrt{N} q \tau)\right) .
\end{aligned}
$$

This completes the proof.

The following corollary is immediately obtained from Corollary 11.

Corollary 12. We use the same notation as in Proposition 10. In the case $k=2$ choose $N$ with

$$
\frac{\sqrt{N}}{(\log (N+1))^{2}} \geq \max \left\{q \tau(\log (q \tau))^{2}, C_{2,2}\right\},
$$

and for $k>2$ choose $N$ with

$$
\frac{\sqrt{N}}{\log (N+1)} \geq \max \left\{q \tau \log (q \tau), C_{2, k}\right\},
$$

where $C_{2, k}$ is a positive constant depending only on $k$. Then

$$
\sum_{f \in \mathcal{F}}|L(f, \chi, 1 / 2+i t)|^{2} \leq 5 \frac{(4 \pi)^{k-1}}{(k-2) !} \log (\sqrt{N} q \tau) .
$$

5. Proof of theorems. First, we prove Theorem 2. By using Cauchy's inequality, it follows that

$$
\begin{aligned}
&\left|\sum_{f \in \mathcal{F}} \overline{a_{f, \infty}(1)} L(f, \chi, 1 / 2+i t)\right|^{2} \\
& \leq \sum_{\substack{f \in \mathcal{F} \\
L(f, \chi, 1 / 2+i t) \neq 0}}\left|a_{f, \infty}(1)\right|^{2} \sum_{f \in \mathcal{F}}|L(f, \chi, 1 / 2+i t)|^{2} .
\end{aligned}
$$


Choosing $N$ as in the statement of Theorem 2, and substituting the estimates of Corollaries 8 and 12 into (25), we obtain the assertion of Theorem 2.

Next, we prove Theorem 3. For $k=2,4,6,8,10,14$ and a prime $p>c$, where $c$ is a positive absolute constant, let $\mathfrak{F}_{k, p}$ denote an orthogonal basis in $S_{k}\left(\Gamma_{0}(p)\right)$ which consists of normalized newforms. We choose $\mathcal{F}$ as

$$
\mathcal{F}=\left\{f /\|f\|: f \in \mathfrak{F}_{k, p},\|f\|=\langle f, f\rangle^{1 / 2}\right\} .
$$

Then, from Theorem 2, we have

$$
\sum_{\substack{\left.f \in \mathfrak{F}_{k, p} \\ f, \chi, 1 / 2+i t\right) \neq 0}}\left|a_{f /\|f\|, \infty}(1)\right|^{2} \gg \frac{(4 \pi)^{k-1}}{(k-2) !} \cdot \frac{1}{\log p} .
$$

Combining (26) with the estimate due to Hoffstein-Lockhart [6] and Goldfeld-Hoffstein-Lieman [5]

$$
\left|a_{f /\|f\|, \infty}(1)\right|^{2} \operatorname{Vol}\left(\Gamma_{0}(p) \backslash \mathfrak{H}\right) \ll \log (k p+1),
$$

where the implied constant is absolute (see Theorem 0.1 of [6] and Main Theorem of [5]), we obtain the assertion of Theorem 3 .

6. An asymptotic formula for the second moment. Fix $q$ and $t$. In this section we shall derive an asymptotic formula for the second moment which is uniform in $N \rightarrow \infty$ by generalizing the method of Duke [3]. It seems that this method is not effective to observe the dependence on $q$ and $t$.

Let $f, g \in S_{k}\left(\Gamma_{0}(N)\right), t$ a real number, and $\chi$ a primitive character (mod $q$ ) with $(N, q)=1$. From the functional equation (6) it follows that

$$
\begin{aligned}
\left(\frac{4 \pi^{2}}{N q^{2}}\right)^{-w} & \Gamma(k / 2+w+i t) \Gamma(k / 2+w-i t) \\
\times & \times(f, \chi, w+1 / 2+i t) L(g, \bar{\chi}, w+1 / 2-i t) \\
= & \left(\frac{4 \pi^{2}}{N q^{2}}\right)^{w} \Gamma(k / 2-w+i t) \Gamma(k / 2-w-i t) \\
& \times L\left(f \mid\left[\sigma_{0}\right]_{k}, \bar{\chi},-w+1 / 2-i t\right) L\left(g \mid\left[\sigma_{0}\right]_{k}, \chi,-w+1 / 2+i t\right) .
\end{aligned}
$$

For $f \in S_{k}\left(\Gamma_{0}(N)\right)$ we define the function $f \mid K$ by

$$
(f \mid K)(z)=\overline{f(-\bar{z})} .
$$

Writing

we have the expression

$$
f(z)=\sum_{n=1}^{\infty} \widehat{a}_{f, \infty}(n) e^{2 \pi i n z},
$$

$$
(f \mid K)(z)=\sum_{n=1}^{\infty} \overline{\widehat{a}_{f, \infty}(n)} e^{2 \pi i n z} .
$$


It is known that $f \mid K \in S_{k}\left(\Gamma_{0}(N)\right)$ and $f|K|\left[\sigma_{0}\right]_{k}=f\left|\left[\sigma_{0}\right]_{k}\right| K$ (see p. 296 of $\mathrm{Li}[8])$. From these properties it follows that

$$
L\left(f|K|\left[\sigma_{0}\right]_{k}, \chi,-w+1 / 2+i t\right)=L\left(f\left|\left[\sigma_{0}\right]_{k}\right| K, \chi,-w+1 / 2+i t\right) .
$$

Therefore, putting $g=f \mid K$ in (27), we obtain the following lemma.

Lemma 13. Let $k$ be a positive even integer, $f \in S_{k}\left(\Gamma_{0}(N)\right), t$ a real number, and $\chi$ a primitive character $(\bmod q)$ with $(N, q)=1$. Then

$$
\begin{aligned}
\left(\frac{4 \pi^{2}}{N q^{2}}\right)^{-w} \Gamma(k / 2+w+i t) \Gamma(k / 2+w-i t) \\
\quad \times L(f, \chi, w+1 / 2+i t) L(f \mid K, \bar{\chi}, w+1 / 2-i t) \\
=\left(\frac{4 \pi^{2}}{N q^{2}}\right)^{w} \Gamma(k / 2-w+i t) \Gamma(k / 2-w-i t) \\
\quad \times L\left(f \mid\left[\sigma_{0}\right]_{k}, \bar{\chi},-w+1 / 2-i t\right) L\left(f\left|\left[\sigma_{0}\right]_{k}\right| K, \chi,-w+1 / 2+i t\right) .
\end{aligned}
$$

The following lemma is an analogue of Lemma 3 of Duke [3].

LEMMA 14. Let $1 / 2<c<1$. Using the same notation as in Lemma 13 , we have

$$
\begin{aligned}
|\Gamma(k / 2+i t)|^{2}|L(f, \chi, 1 / 2+i t)|^{2} & \\
= & \sum_{m=1}^{\infty} \sum_{n=1}^{\infty} \frac{\chi(m) \overline{\chi(n)} a_{f, \infty}(m) \overline{a_{f, \infty}(n)}}{(m n)^{1 / 2}}\left(\frac{n}{m}\right)^{i t} G(m, n, t) \\
& +\sum_{m=1}^{\infty} \sum_{n=1}^{\infty} \frac{\overline{\chi(m)} \chi(n) a_{f, 0}(m) \overline{a_{f, 0}(n)}}{(m n)^{1 / 2}}\left(\frac{m}{n}\right)^{i t} G(m, n, t)
\end{aligned}
$$

with

$$
G(m, n, t)=\frac{1}{2 \pi i} \int_{(c)}\left(\frac{4 \pi^{2} m n}{N q^{2}}\right)^{-w} \frac{\Gamma(k / 2+w+i t) \Gamma(k / 2+w-i t)}{w} d w .
$$

Pr o of. From (28) it follows that

$$
\begin{aligned}
& \frac{1}{2 \pi i} \int_{(c)}\left(\frac{4 \pi^{2}}{N q^{2}}\right)^{-w} \frac{\Gamma(k / 2+w+i t) \Gamma(k / 2+w-i t)}{w} \\
& \quad \times L(f, \chi, w+1 / 2+i t) L(f \mid K, \bar{\chi}, w+1 / 2-i t) d w \\
& =\sum_{m=1}^{\infty} \sum_{n=1}^{\infty} \frac{\chi(m) \overline{\chi(n)} a_{f, \infty}(m) \overline{a_{f, \infty}(n)}}{(m n)^{1 / 2}}\left(\frac{n}{m}\right)^{i t} G(m, n, t) .
\end{aligned}
$$

By moving the contour of integration to the vertical line $\Re w=-c$, we find that the left-hand side of (29) equals 


$$
\begin{aligned}
\Gamma(k / 2+ & i t) \\
\times & \Gamma(k / 2-i t) \\
& +\frac{1}{2 \pi i} \int_{(-c)}\left(\frac{4 \pi^{2}}{N q^{2}}\right)^{-w} \frac{\Gamma(k / 2+w+i t) \Gamma(k / 2+w-i t)}{w} \\
& \times L(f, \chi, w+1 / 2+i t) L(f \mid K, \bar{\chi}, w+1 / 2-i t) d w .
\end{aligned}
$$

The first term in (30) is equal to $|\Gamma(k / 2+i t)|^{2}|L(f, \chi, 1 / 2+i t)|^{2}$, because of $L(f \mid K, \bar{\chi}, 1 / 2-i t)=\overline{L(f, \chi, 1 / 2+i t)}$. From Lemma 13 the second term in $(30)$ is

$$
\begin{aligned}
\frac{1}{2 \pi i} & \int_{(-c)}\left(\frac{4 \pi^{2}}{N q^{2}}\right)^{w} \frac{\Gamma(k / 2-w+i t) \Gamma(k / 2-w-i t)}{w} \\
& \times L\left(f \mid\left[\sigma_{0}\right]_{k}, \bar{\chi},-w+1 / 2-i t\right) L\left(f\left|\left[\sigma_{0}\right]_{k}\right| K, \chi,-w+1 / 2+i t\right) d w,
\end{aligned}
$$

and, by changing variables $w$ to $-w$, this equals

$$
-\sum_{m=1}^{\infty} \sum_{n=1}^{\infty} \frac{\overline{\chi(m)} \chi(n) a_{f, 0}(m) \overline{a_{f, 0}(n)}}{(m n)^{1 / 2}}\left(\frac{m}{n}\right)^{i t} G(m, n, t) .
$$

This completes the proof.

Proposition 15. Let $k$ be a positive even integer, $\mathcal{F}$ an orthonormal basis in $S_{k}\left(\Gamma_{0}(N)\right)$, and $\chi$ a primitive character $(\bmod q)$ with $(N, q)=1$. For any $\varepsilon>0$ we have

$$
\begin{aligned}
\sum_{f \in \mathcal{F}}|L(f, \chi, 1 / 2+i t)|^{2}= & \frac{(4 \pi)^{k-1}}{(k-2) !} \cdot \frac{\phi(q)}{q} \log N+C_{k, q, t} \\
& +O\left(N^{(1-k) / 2+\varepsilon}\right), \quad N \rightarrow \infty
\end{aligned}
$$

where $\phi(\cdot)$ is the Euler function, $C_{k, q, t}$ is a computable constant, and the implied constant depends on $k, q, t$, and $\varepsilon$.

P r o of. Summing up the formula of Lemma 14 over $\mathcal{F}$ and using Lemma 5(i), we have

$$
\begin{aligned}
|\Gamma(k / 2+i t)|^{2} & \sum_{f \in \mathcal{F}}|L(f, \chi, 1 / 2+i t)|^{2} \\
= & 2 \frac{(4 \pi)^{k-1}}{(k-2) !} \sum_{n=1}^{\infty} \frac{\chi_{0}(n)}{n} G(n, n, t) \\
& +O\left(N^{1 / 2-k} d(N) \sum_{m=1}^{\infty} \sum_{n=1}^{\infty} \frac{|G(m, n, t)|(m, n)^{1 / 2}}{(m n)^{-k / 2+1}}\right)
\end{aligned}
$$


where $\chi_{0}$ is the principal character $\bmod q$. To estimate the error term we use

$$
\begin{aligned}
|G(m, n, t)| & =\left|\frac{1}{2 \pi i} \int_{\left(c^{\prime}\right)}\left(\frac{4 \pi^{2} m n}{N q^{2}}\right)^{-w} \frac{\Gamma(k / 2+w+i t) \Gamma(k / 2+w-i t)}{w} d w\right| \\
& \ll\left(\frac{m n}{N q^{2}}\right)^{-c^{\prime}}
\end{aligned}
$$

for any $c^{\prime}>0$. Then the error term in (31) is

$$
\ll N^{1 / 2-k+c^{\prime}} d(N) q^{2 c^{\prime}} \sum_{m=1}^{\infty} \sum_{n=1}^{\infty} \frac{(m, n)^{1 / 2}}{(m n)^{c^{\prime}-k / 2+1}} .
$$

Since the double sum in (32) is convergent for $c^{\prime}>k / 2$, we specify $c^{\prime}=$ $k / 2+\varepsilon$ to get

$$
\ll N^{(1-k) / 2+\varepsilon} d(N) q^{k+\varepsilon} \text {. }
$$

Next, we have

$$
\begin{aligned}
\sum_{n=1}^{\infty} \frac{\chi_{0}(n)}{n} G(n, n, t)= & \frac{1}{2 \pi i} \int_{(c)}\left(\frac{4 \pi^{2}}{N q^{2}}\right)^{-w} L\left(2 w+1, \chi_{0}\right) \\
& \times \frac{\Gamma(k / 2+w+i t) \Gamma(k / 2+w-i t)}{w} d w
\end{aligned}
$$

where $L(\cdot, \chi)$ is the Dirichlet $L$-function attached to $\chi$, and moving the contour of integration to the vertical line $\Re w=-d$ with $1 / 2<d<k / 2$ shows that this equals

$$
\begin{aligned}
\operatorname{Res}_{w=0} & \left\{\left(\frac{4 \pi^{2}}{N q^{2}}\right)^{-w} L\left(2 w+1, \chi_{0}\right) \frac{\Gamma(k / 2+w+i t) \Gamma(k / 2+w-i t)}{w}\right\} \\
& +\frac{1}{2 \pi i} \int_{(-d)}\left(\frac{4 \pi^{2}}{N q^{2}}\right)^{-w} L\left(2 w+1, \chi_{0}\right) \\
& \times \frac{\Gamma(k / 2+w+i t) \Gamma(k / 2+w-i t)}{w} d w .
\end{aligned}
$$

Using the expression $L\left(s, \chi_{0}\right)=\prod_{p \mid q}\left(1-1 / p^{s}\right) \zeta(s)$, where $\zeta(s)$ is the Riemann zeta-function, and further using the functional equation for $\zeta(s)$, we see that the second term in (34) is

$$
\begin{aligned}
& \ll N^{-d} \int_{(-d)}\left|\frac{\Gamma(-w)}{\Gamma(w+1 / 2)} \cdot \frac{\Gamma(k / 2+w+i t) \Gamma(k / 2+w-i t)}{w}\right||d w| \\
& \ll N^{-d},
\end{aligned}
$$


which is equal to $N^{(1-k) / 2-\varepsilon}$ by putting $d=(k-1) / 2+\varepsilon$. The first term in (34) equals

$$
\frac{1}{2} \cdot \frac{\phi(q)}{q}|\Gamma(k / 2+i t)|^{2} \log N+C_{k, q, t}^{\prime}
$$

where $C_{k, q, t}^{\prime}$ is some computable constant. Hence

$$
\begin{aligned}
\sum_{n=1}^{\infty} \frac{\chi_{0}(n)}{n} G(n, n, t)= & \frac{1}{2} \cdot \frac{\phi(q)}{q}|\Gamma(k / 2+i t)|^{2} \log N+C_{k, q, t}^{\prime} \\
& +O\left(N^{(1-k) / 2-\varepsilon}\right) .
\end{aligned}
$$

Substituting (33) and (35) into (31), we now obtain the assertion of this proposition.

REMARK. In (33) we are concerned with the dependence on $q$ as compared to that in Corollary 12. To insure $N^{(1-k) / 2} q^{k} \leq 1$ in (33) we need to choose $N$ so that $\sqrt{N} \geq q^{1+1 /(k-1)}$. This is worse than the lower bound condition in Corollary 12.

The implied constant in Proposition 15 depends exponentially on $t$.

Note. After this paper was accepted for publication, I found a recent paper by A. Akbary, Non-vanishing of weight $k$ modular L-functions with large level, J. Ramanujan Math. Soc. 14 (1999), 37-54. In that paper a generalization of Duke's result to newforms of even weight $k$ and prime level $N$ has been shown.

Added in proof (March 2000). Refinements of Duke's result, which assert that the proportion of non-vanishing forms are indeed positive, have been shown by J. Vanderkam, The rank of quotients of $J_{0}(N)$, Duke Math. J. 97 (1999), 545-577, and E. Kowalski and P. Michel, The analytic rank of $J_{0}(q)$ and zeros of automorphic L-functions, Duke Math. J. 100 (1999), 503-542, independently of each other.

\section{References}

[1] R. Balasubramanian and K. Ramachandra, An alternative approach to a theorem of Tom Meurman, Acta Arith. 55 (1990), 351-364.

[2] F. Chamizo and J. Pomykała, Distribution of zeros of twisted automorphic L-functions with large level, Math. Z. 229 (1998), 575-588.

[3] W. Duke, The critical order of vanishing of automorphic L-functions with large level, Invent. Math. 119 (1995), 165-174.

[4] W. Duke, J. B. Friedlander and H. Iwaniec, Bounds for automorphic Lfunctions, II, ibid. 115 (1994), 219-239.

[5] D. Goldfeld, J. Hoffstein and D. Lieman, An effective zero-free region, Appendix to: Coefficients of Maass forms and the Siegel zero, Ann. of Math. 140 (1994), $177-181$. 
[6] J. Hoffstein and P. Lockhart, Coefficients of Maass forms and the Siegel zero, ibid. 140 (1994), 161-176.

[7] H. Iwaniec, Topics in Classical Automorphic Forms, Grad. Studies Math. 17, Amer. Math. Soc., Providence, RI, 1997.

[8] W. W. Li, Newforms and functional equations, Math. Ann. 212 (1975), 285-315.

[9] G. Shimura, Introduction to the Arithmetic Theory of Automorphic Functions, Princeton Univ. Press, 1971.

[10] A. Weil, On some exponential sums, Proc. Nat. Acad. Sci. U.S.A. 34 (1948), 204207.

Graduate School of Mathematics

Nagoya University

Chikusa-ku, Nagoya 464-8602

Japan

E-mail: m95011@math.nagoya-u.ac.jp 